\title{
IBD Patients Show a Unique Skin Microbiota Composition and a Correlation Pattern Between Serological Biomarkers and Clinical Aspects During Skin Adverse Events in the Setting of Anti-TNFa Therapy
}

\section{Zuzana Stehlikova}

Institute of Microbiology of the Czech Academy of Sciences, Prague

Filip Rob

Department of Dermatovenerology, Second Faculty of Medicine, Charles University, University Hospital Bulovka, Prague

\section{Martin Kolar}

IBD Clinical and Research Centre ISCARE a.s., Prague

\section{Dagmar Schierova}

Institute of Microbiology of the Czech Academy of Sciences, Prague Jakub Kreisinger

Department of Zoology, Faculty of Science, Charles University, Prague

\section{Zuzana Jackova}

Institute of Microbiology of the Czech Academy of Sciences, Prague

\section{Radka Roubalova}

Institute of Microbiology of the Czech Academy of Sciences, Prague

\section{Stepan Coufal}

Institute of Microbiology of the Czech Academy of Sciences, Prague

\section{Martin Mihula}

Institute of Microbiology of the Czech Academy of Sciences, Prague

\section{Tomas Thon}

Institute of Microbiology of the Czech Academy of Sciences, Prague

\section{Lukas Bajer}

Institute of Clinical and Experimental Medicine, Hepatogastroenterology, Prague

\section{Michaela Novakova}

Department of Dermatovenerology, Second Faculty of Medicine, Charles University, University Hospital Bulovka, Prague

Martin Vasatko 
Department of Dermatovenerology, Second Faculty of Medicine, Charles University, University Hospital Bulovka, Prague

\section{Klara Kostovcikova}

Institute of Microbiology of the Czech Academy of Sciences, Prague

\section{Natalie Galanova}

Institute of Microbiology of the Czech Academy of Sciences, Prague

\section{Milan Lukas}

IBD Clinical and Research Centre ISCARE a.s., Prague

\section{Miloslav Kverka}

Institute of Microbiology of the Czech Academy of Sciences, Prague

\section{Jana Hercogova}

Department of Dermatovenerology, Second Faculty of Medicine, Charles University, University Hospital Bulovka, Prague

\section{Helena Tlaskalova-Hogenova}

Institute of Microbiology of the Czech Academy of Sciences, Prague

Zuzana Jiraskova Zakostelska ( $\square$ zakostelska@biomed.cas.cz )

Institute of Microbiology of the Czech Academy of Sciences, Prague

\section{Research Article}

Keywords: skin microbiota, IBD, anti-TNFa, 16S rRNA gene sequencing, serum biomarkers, skin adverse events

Posted Date: March 7th, 2022

DOI: https://doi.org/10.21203/rs.3.rs-1415112/v1

License: (c) (1) This work is licensed under a Creative Commons Attribution 4.0 International License. Read Full License

Version of Record: A version of this preprint was published at Frontiers in Cellular and Infection Microbiology on January 1st, 2023. See the published version at https://doi.org/10.3389/fcimb.2022.1064537. 


\section{Abstract \\ Background}

Crohn's disease (CD) and ulcerative colitis (UC) are two forms of inflammatory bowel disease (IBD), where gut but not skin dysbiosis is well recognized. Tumor-necrosis factor alpha (TNFa) blockers have been successful in IBD treatment, but up to a quarter of patients suffer from skin adverse events (SkAE), for which we do not have a tool to predict them. Therefore, we examined the skin microbiota composition of IBD patients at several body sites by $16 \mathrm{~S}$ bacterial profiling. Moreover, we quantified 22 serum markers of inflammation and epithelial barrier disruption in the setting of anti-TNFa therapy and SkAE manifestation by ELISA.

\section{Results}

The skin microbiota signature of IBD patients differed markedly from healthy subjects. In particular, skin microbiota of $\mathrm{CD}$ patients differed significantly from that of $\mathrm{UC}$ and $\mathrm{HC}$ at the retroauricular crease. $\mathrm{CD}$ patients showed high abundance of Corynebacterium (ASV86) and lower abundance of Actinomyces (ASV28), Cutibacterium (ASV212), Lawsonella (ASV87), and other taxa compared to HC. UC patients showed higher abundance of Delftia (ASV1039) and Pseudomonas (ASV1183), and lower abundance of Actinomyces (ASV28), Streptococcus (ASV607), or Haemophilus (ASV1150) than HC. Although microbiome changes were correlated at the ASV level in CD and UC patients compared to HC, we identified some bacterial ASV's that exhibited changes typical of individual IBD forms. Manifestation of SkAE during anti-TNFa therapy was associated with a specific microbiota profile on the skin, and with a decrease in serum levels of L-FABP and I-FABP.

\section{Conclusions}

The overall proinflammatory effect of IBD might predispose for altered skin microbiota composition, hence promoting SkAE manifestation during anti-TNFa therapy. We identified specific serum markers associated with the incidence of SkAE during anti-TNFa therapy, however, further studies are needed to shed light on the impact of microbiota in this matter. Combining sequencing technology with search for serum biomarkers has opened up new opportunities in studying SkAE related to anti-TNFa therapy. Early detection of changes in microbiota-host equilibrium may have beneficial consequences in revealing the pathogenesis of SkAE in IBD.

\section{Background}

The skin and mucosal surfaces are the main barriers preventing excessive contact with the external environment, and their interconnection is well established and referred to as the "gut-skin axis". Gut microbiota alterations, impaired intestinal barrier integrity, or side effects of various drugs often manifest 
on the skin. The skin and its microbiota are largely neglected in many inflammatory conditions, yet they may be useful aspects to include in the search for disease risk factors and they may even serve as predictors of adverse events of anti-tumor-necrosis alpha (TNFa) treatment in inflammatory bowel disease (IBD).

IBD has two most prevalent forms, Crohn's disease (CD) and ulcerative colitis (UC). IBD prevalence has been steadily increasing since 1990, reaching as high as $0.5 \%$ in Western Europe, including the Czech Republic [1,2]. Despite the unknown etiology and exact pathogenesis of IBD, it is generally accepted that one of the main triggers is the aberrant immune response to commensal gut microbiota antigens in genetically susceptible individuals [3]. Up to $40 \%$ of IBD patients suffer from extra-intestinal manifestations affecting various organs, including the skin [4]. Skin manifestations of IBD can share the same histological features with manifestations in the gastrointestinal tract, e.g., granulomatous cutaneous lesions [5], and can arise due to immune mechanisms triggered by common antigens shared by gut bacteria and the skin [6].

TNFa is a key pleiotropic cytokine involved in the pathogenesis of many inflammatory diseases, including IBD. More than two decades ago, the first anti-TNFa agent was approved for the treatment of IBD and since then, TNFa blockers have been widely used [7]. Interestingly, around $20 \%$ of IBD patients treated with anti-TNFa develop skin adverse events (SkAE) such as eczema or psoriasiform dermatitis, referred to as paradoxical reactions [8-11]. Surprisingly, despite the widespread use of TNFa blockers in IBD therapy, it is still poorly understood how the blocking of TNFa influences the development of skin adverse events in IBD patients. In this case, serum biomarkers are indispensable tools which not only extent our knowledge about pathogenic mechanisms of the disease, but also reflect the spectrum of disease manifestation. Serum biomarkers are particularly important where both skin and gut are affected in the disease pathogenesis or manifestation. For example, fatty acid binding proteins, which are serum biomarkers of inflammation and epithelial barrier dysfunction, are important pathogenic factors in both skin and gut involvement, and they may serve as early predictors of SkAE following anti-TNFa treatment.

Many studies are mainly focused on gut microbiota analysis in IBD patients. These studies showed that levels of Faecalibacterium prausnitzii are decreased and the abundance of Veillonella and Escherichia coli increased in CD patients. UC patients showed decreased abundance of Eubacterium rectale, Akkermansia muciniphila, and Roseburia and an increase in Escherichia coli $[12,13]$. Such changes in gut microbial communities can provide an opportunity for certain microbes to spread, changing thus the overall physiological setting of the microbial community. Altered behavior of microbial species and an abnormal, excessive immune response to the overgrown microbes can disrupt microbiota-mucosa interactions, allowing antigens to penetrate the mucosal barrier. This may further stimulate local and systemic immunity $[14,15]$ and promote the release of mediators such as lipopolysaccharide-binding protein, matrix metalloproteinases, defensins, or fatty acid-binding proteins [16-19]. A dysbiotic intestinal environment thus can be responsible for the global increase in IBD incidence and its frequent extraintestinal manifestation on the skin [4, 20-24]. In addition, the proinflammatory effect of IBD can also 
promote changes in skin microbiota composition. Specific composition of skin microbiota can then predispose for SkAE manifestation following anti-TNFa treatment in IBD patients.

In this pilot study, we characterized the composition of the skin microbiota of CD and UC patients. Furthermore, we analyzed the temporal dynamics of the skin microbiota and serum biomarkers of inflammation and epithelium barrier dysfunction during anti-TNFa treatment to uncover any links to the subsequent development of SkAE.

\section{Materials And Methods}

\section{Patients and Sample Collection}

For our pilot study, 87 IBD patients were recruited at the ISCARE Clinical and Research Center for Inflammatory Bowel Disease and their skin was subsequently examined at the University Hospital Bulovka in the Czech Republic from November 2018 to December 2020. 41 healthy control subjects (HC) were recruited at the Institute of Clinical and Experimental Medicine (IKEM) from November 2018 to December 2020.

Patients were diagnosed according to the European Crohn's and Colitis Organization (ECCO) guidelines [25]. Patients indicated for / already receiving anti-IBD biologic therapy, such as anti-TNFa or anti IL$12 / 23$ were included in the study. Only patients who were indicated for biologic treatment initiation with anti-TNFa, i.e., patients who neither received any biologic treatment nor were prior exposed to anti-TNFa, were recruited for the part of study monitoring the development of SkAE. In all patients, we recorded their medical history, disease duration, comorbidities, concomitant immunosuppression, response to the treatment, and various clinical and laboratory parameters at every patients' visit (at baseline and at weeks $4,8,16,22,32$, and 38 - the study endpoint) so that we could monitor the development of SkAE in antiTNFa-treated patients over time. The study endpoint refers to the end of sample collection, not to the SkAE healing. The following clinical parameters were monitored: Harvey-Bradshaw index (HBI) in patients with CD and partial Mayo score (pMayo) in patients with UC. HBI or pMayo were calculated by specialist gastroenterologists during patients' visits based on clinical disease activity reflected by the patients' reported outcomes and the physicians' assessment $[26,27]$. The following laboratory parameters were measured: C-reactive protein (CRP); fecal calprotectin (FC); ferritin; hemoglobin ( $\mathrm{Hb}$ ); platelets (PLT); white blood cells (WBC). The exclusion criteria for IBD patients were the use of antibiotics within 3 months prior sampling, prior dermatologic diseases or dermatologic extraintestinal manifestation of IBD. 
Table 1

Overview of IBD patients and healthy controls parameters included in the sequencing analysis at the time of their recruitment. The values are shown as median with the minimum and maximum values in parentheses.

\begin{tabular}{|llll|}
\hline \multicolumn{3}{|l|}{ IBD patients $(\mathrm{n}=\mathbf{4 0})$} & Healthy controls \\
\hline & $\mathrm{CD}(\mathrm{n}=30)$ & $\mathrm{UC}(\mathrm{n}=10)$ & $\mathrm{HC}(\mathrm{n}=25)$ \\
\hline Female $(\mathrm{n})$ & 22 & 7 & 16 \\
\hline Male $(\mathrm{n})$ & 8 & 3 & 9 \\
\hline Age & $35(19,60)$ & $34.5(22,63)$ & $34.5(20,47)$ \\
\hline BMI & $23.41(17.80,35.26)$ & $20.97(17.30,25.95)$ & $22.72(18.5,33.51)$ \\
\hline Disease duration (years) & $6(1,24)$ & $9(1,20)$ & - \\
\hline Age at diagnosis & $27(6,55)$ & $27(17,49)$ & - \\
\hline HBI & $3(0,10)$ & - & - \\
\hline pMayo & - & $7(2,12)$ & - \\
\hline
\end{tabular}

Out of the 87 patients, 47 patients were not included in the study mainly because of primary nonresponse to anti-TNFa therapy, loss of venous access, frequent nasopharyngeal infections, or due to the current COVID-19 pandemic situation. The exclusion criteria for healthy subjects were a gastrointestinal or dermatological diagnosis, concurrent or previous biologic therapy, and the use of antibiotics within 3 months before sampling. A total of 40 patients and 25 healthy controls were included in the sequencing analysis. For the analysis of skin microbiota composition in SkAE, we used 25 IBD patients on anti-TNFa treatment who provided the complete sample series necessary for the longitudinal analysis, i.e., whose baseline samples were not influenced by prior exposure to any biologics. The clinical and laboratory data and baseline characteristics for all patients and controls at the time of their recruitment are summarized in Table 1; for a detailed overview of the characteristics of patients selected for longitudinal observation, see Supplementary Table S1. All study participants signed informed consent forms. This study was approved by the Ethics Committee of ISCARE (Nr2017/Ila), IKEM (G 17-06-09), and University Hospital Bulovka (21.6.2017 I8513IEK-Z).

Samples were collected at each patients' visit to capture the SkAE incidence. Blood samples were collected as previously described [15]. Serum was collected at each patients' visit and stored at $-80^{\circ} \mathrm{C}$ until analysis. We selected serum biomarkers associated with skin and gut barrier function (e.g., E-FABP (Epidermal fatty acid-binding protein), L-FABP (Liver fatty acid-binding protein), and I-FABP (Intestinal fatty acid-binding protein)), antimicrobial response (e.g., S100A8), and inflammation (e.g., IL-18, TNFa) and we quantified them using ELISA (Supplementary Table S2) [15]. All ELISA tests were performed according to the manufacturers' instructions. We used paired t-test to compare two experimental groups or one-way analysis of variance (ANOVA) with Dunnett's multiple comparison test to compare multiple 
groups. Differences were considered statistically significant at $p \leq 0.05$. Correlation analysis of selected clinical factors and biomarkers was applied and we adjusted $p$-values by false discovery rate. Correlation data were displayed using Spearman correlation coefficient. Skin swab samples were collected at the University Hospital Bulovka (Prague, Czech Republic). Patients were instructed not to use antiseptics for 7 days before sampling and not to bathe or wash the specific skin sites for 24 hours before sampling. Samples were obtained from a $4 \mathrm{~cm}^{2}$ area using a sterile flocked swab (FLOQSwabsTM COPAN Diagnostics INC., USA) soaked in sterile SCF-1 buffer, as previously described [28, 29]. All samples were stored at $-80^{\circ} \mathrm{C}$ until further processing. Sites where SkAE associated with anti-TNFa treatment usually occur were sampled, namely the flexor regions - elbow and knee, retroauricular crease, skin under the lower eyelid, lumbar area, inguinal crease, and genitalia. The regions meeting the minimum sample size requirements (the threshold was set to 30 based on the amounts of particular samples we obtained), i.e., the retroauricular crease (198), the lumbar area (112), and the inguinal crease (30) were ultimately processed for further analysis (for a more detailed information on sample collection please see Supplementary Figure S1).

\section{Skin Microbiota Analysis}

Extraction of total DNA from swabs was performed using the DNeasy PowerBiofilm kit (Qiagen, Germany) with minor modifications to the protocol as previously described [29, 30]. The subsequent PCR amplification of bacterial DNA was performed with degenerate primers $341 \mathrm{~F}$ and $806 \mathrm{R}$, which target the V3V4 region of 16S rRNA, as previously described [31]. Briefly, a $25 \mu$ reaction mixture was prepared for each sample in triplicates. PCR amplification was performed using $1 \mathrm{X}$ HiFi polymerase (Roche, United States), $0.4 \mu \mathrm{M}$ primers, and $5 \mu$ l of template. Thermal cycling parameters were 33 cycles of denaturation $\left(94^{\circ} \mathrm{C}, 3 \mathrm{~min}\right)$, annealing $\left(55^{\circ} \mathrm{C}, 5 \mathrm{~s}\right)$, and extension $\left(72^{\circ} \mathrm{C}, 2 \mathrm{~min}\right)$. Triplicates of PCR products were pooled to minimize random PCR bias, and the correct length of amplicons was verified by agarose gel electrophoresis. PCR amplification negative controls, extraction and sequencing positive controls (mock communities; ZymoBIOMICS Microbial Community Standard and ZymoBIOMICS Microbial Community DNA Standard, Zymo Research, USA; both in linear and logarithmic form) were processed in a similar manner.

The PCR amplicons were processed as previously described [29]. Briefly, the pooled amplicons were normalized using a Sequal-PrepTM Normalization Plate (96) Kit (Illumina, United States). Adapters compatible with the MiSeq platform were ligated using the KAPA HyperPrep kit (Roche, United States), quantified, and sequenced using the MiSeq Reagent Kit v2 (2 × $300 \mathrm{bp})$ at the CEITEC Genomics Core Facility (Brno, Czech Republic). The raw sequence data are available in the Sequence Read Archive (SRA) under the accession number SUB10259106 and BioProject ID PRJNA757573.

Sequences were quality filtered with Cutadapt (version 1.15), joined with Fastq-join (1.3) and demultiplexed. The samples were rarefied at minimal sequencing depth which was 3415 . The amplicon sequence variants (ASVs) were generated using DADA2, and taxonomy was assigned by vsearch against 
the SILVA database (release 138) with $99 \%$ similarity. Diversity analysis was performed in Qiime2 version 2020.11 (37). Scripts used for Qiime2 diversity and taxonomic analyses, and ASV table are part of the supplementary material (Supplementary scripts.zip).

Variation in alpha diversity was analyzed using Linear Mixed Models (LMM) with alpha diversity indices as the response variable (Chao1 and number of detected ASVs were log10-transformed) and patients' identity as a random effect. Week after treatment initiation and body mass index were included as a continuous predictor, whereas sex and diagnosis were included as categorical predictors. Separate LMM was fitted for each sampling site. The significance of predictors was tested using likelihood tests and Tukey post-hoc comparisons. The models were fitted using the R package Ime4 [32]. Beta diversity was presented in principle coordinate analysis (PCoA) plots and assessed using unweighted UniFrac and Bray Curtis distances. Permutational multivariate analysis of variance (PERMANOVA) was used to confirm statistical significance between $\mathrm{CD}, \mathrm{UC}$, and healthy controls. Mixed models for longitudinal data from the $\mathrm{R}$ package metamicrobiome $\mathrm{R}$ with individual identity as a random factor were used to examine the bacterial taxa involved in the microbiome changes between $\mathrm{CD}, \mathrm{UC}$ and the HC group [33]. ASVs present in $<10 \%$ of the samples were not included in these analyzes to avoid spurious results. MetamicrobiomeR package was also used for differential abundance analysis of $\mathrm{CD}^{+} \mathrm{vs} \mathrm{CD}^{-}$and $\mathrm{UC}^{+}$vs $\mathrm{UC}^{-}$samples (i.e., samples of patients who either developed (+) or not developed (-) SkAE during anti-TNFa treatment) (33).

\section{Results}

\section{Skin Microbiota Diversity Distinguishes Cd And Uc Patients}

To find out whether the skin microbiota differs between CD and UC patients and healthy controls (HC), we analyzed the bacterial composition of the skin at the retroauricular crease, the lumbar region, and the inguinal crease using amplicon 16S rRNA gene sequencing.

Separate models for each sampling site and diagnosis revealed that microbiota at the retroauricular crease of $\mathrm{CD}$ patients has significantly increased Shannon index (richness and evenness) compared to $\mathrm{UC}$ or $\mathrm{HC}$, while the difference between $\mathrm{UC}$ and $\mathrm{HC}$ was not pronounced. The number of amplicon sequence variants (ASVs) and Chao1 index showed only slightly increased richness and evenness of the skin microbiota at the retroauricular crease in CD patients compared to UC patients and HC (Fig. 1A). Beta diversity of skin microbial communities at the reatroauricular crease, expressed as Unweighted UniFrac and Bray-Curtis distance between $C D, U C$, and HC showed that samples cluster by diagnosis. As a result, $\mathrm{UC}$ and $\mathrm{HC}$ were shown to be more similar to each other (Fig. 1B). We confirmed that these differences were not influenced by biologic treatment, sex, timescale of sampling, BMI, or the number of weeks after treatment initiation (data not shown).

Bacterial microbiota of the skin at retroauricular crease was dominated by the phyla Actinobacteria and Firmicutes, and, to a lesser extent, Proteobacteria. The most abundant bacterial genera in all samples were Cutibacterium, Staphylococcus, and Corynebacterium, followed by the species Staphylococcus 
hominis and Staphylococcus epidermidis. The relative abundance of each taxon differed between CD, UC, and $\mathrm{HC}$, with $\mathrm{CD}$ having a relatively more diverse composition than UC or $\mathrm{HC}$ (Fig. 1C).

We observed slightly higher alpha diversity in CD compared to UC patients, and significantly higher diversity in $\mathrm{CD}$ over $\mathrm{HC}$ at the lumbar region, but not at the inguinal crease (Supplementary Figure S2A and S2B, respectively). We again confirmed that these differences were neither influenced by biologic treatment, sex, timescale of sampling, BMI, nor the number of weeks after treatment initiation (data not shown). Beta diversity of skin microbial communities at the lumbar region showed a significantly distinct microbiota profile of IBD patients when compared to healthy individuals. At the inguinal crease, microbial beta diversity of $\mathrm{CD}$ patients did not differ from $\mathrm{HC}$ but differed from that of UC patients. Beta diversity of UC patients at the inguinal crease was significantly different from that of HC (Supplementary Figure $\mathrm{S} 2 \mathrm{C})$.

Out of the three skin sites we sampled, the retroauricular crease showed the most pronounced differences in skin microbiota composition between $\mathrm{CD}, \mathrm{UC}$, and HC. For that reason, we analyzed this particular sampling site in more detail.

\section{CD and UC have their own microbial pattern while retaining the correlation of the shared taxa}

To explain patterns distinguishing $\mathrm{CD}, \mathrm{UC}$, and $\mathrm{HC}$ that we observed by looking at alpha and beta diversity of our samples, we performed Differential abundance analysis (DAA). We revealed that CD patients had significantly higher abundance of Corynebacterium (ASV86) and Pseudomonas (ASV1183), and significantly lower abundance of other species such as Actinomyces (ASV28), Cutibacterium (ASV212), Lawsonella (ASV87), Prevotella (ASV303) or Streptococcus (ASV607) over HC. UC patients showed increased abundance of Corynebacterium (ASV86) and Pseudomonas (ASV1183) over HC, otherwise they followed similar pattern as CD (Fig. 2A).

The DAA regression coefficients for $\mathrm{CD}$ vs. $\mathrm{HC}$ and UC vs. $\mathrm{HC}$ comparisons were closely correlated (Spearman's correlation: rho $=0.7560, p<0.0001$ ), suggesting that both IBD conditions promote similar responses in the skin microbiota. At the same time, however, additional DAA comparison between the CD and $\mathrm{HC}$ groups showed that each for of IBD possess specific patterns of changes in ASVs abundances. For example, Corynebacterium (ASV86), Bacteroides (ASV278), or Methylobacterium methylorubrum (ASV909) were significantly increased in CD over UC, while Cutibacterium (ASV212), Delftia (ASV1039) or Psychrobacter (ASV1174) were significantly increased in UC over CD (Fig. 2B).

\section{A specific skin microbiota profile might predispose IBD patients for skin side effects manifestation following anti-TNFa treatment}

In this section focused on monitoring SkAE following anti-TNFa treatment, we used longitudinally collected samples from 25 IBD patients (17 CD and $8 \mathrm{UC}$ ). In this study cohort, SkAE at several skin sites affected a total of $13 \mathrm{CD}$ and $4 \mathrm{UC}$ patients. Different SkAE manifestations on different body sites included drug exanthema, eczema, papulopustular exanthema, herpes, and shingles. Comparing all CD 
and UC samples regardless of the sampled site and longitudinal nature of their collection, we observed that skin microbiota of CD patients with SKAE tended to have different proportional composition from that of CD patients without SkAE. Specifically, CD patients with SkAE had lower frequency of Cutibacterium (ASV212) and Staphylococcus (ASV636) and a higher frequency of Corynebacterium (ASV86), Micrococcus (ASV179), Enhydrobacter (ASV1167) or Anaerococcus (ASV757) when compared to $C D$ patients without SkAE. There were no pronounced differences between UC patients with and without SkAE (Supplementary Figure S3A).

As evaluating skin microbiota on several body sites together might be misleading, we further focused on SkAE manifestation (and therefore microbiota comparison) at the retroauricular crease, as this site showed the most pronounced differences in Shannon entropy between CD, UC, and HC. Longitudinally collected samples are particularly useful here, as they could help predict shifts in the microbial composition associated with SkAE manifestation at this skin site. We obtained samples from $2 \mathrm{CD}$ patients with drug exanthema, 2 CD patients with eczema, and 4 UC patients with papulopustular exanthema at the retroauricular crease (Fig. 3A). We did not find any apparent skin microbiota changes that would correspond to SkAE manifestation, IBD severity, or response to the biologic treatment (i.e., loss of response during the treatment), regardless of the metric used (Fig. 3B, 3C, and 3D).

On the other hand, comparing the baseline of SkAE patients $\left(\mathrm{CD}^{+} / \mathrm{UC}^{+}\right)$with the patients who did not go on to develop SkAE $\left(\mathrm{CD}^{-} / \mathrm{UC}^{-}\right)$indicated a potential to predict the development of SkAE based on specific microbiota composition. Percentual taxonomic differences of ASVs between $\mathrm{CD}^{+} / \mathrm{UC}^{+}$and $\mathrm{CD}^{-} / \mathrm{UC}^{-}$ patients and $\mathrm{HC}$ showed that in $\mathrm{CD}$ patients who later developed $\mathrm{SkAE}\left(\mathrm{CD}^{+}\right)$the most abundant genus was Staphylococcus (ASV636) (29\%), whereas in CD patients who did not develop SkAE (CD'), the most abundant genus was Cutibacterium (ASV212) (25\%). Furthermore, in contrast to the $\mathrm{CD}^{-}$cohort, the $\mathrm{CD}^{+}$ cohort was characterized by low abundance of Staphylococcus hominis (ASV642) $\left(2 \% \mathrm{CD}^{+} ; 14 \% \mathrm{CD}^{-}\right)$, higher abundance of Staphylococcus epidermidis (ASV640) $\left(10 \% \mathrm{CD}^{+} ; 5 \% \mathrm{CD}^{-}\right)$, and presence of Dietzia ( $4 \%$ in $C D^{+}$, below $1 \%$ in $C D^{-}$). On the other hand, $C D^{+}$cohort showed only negligible presence of Anaerococcus (ASV757) (below $1 \%$ in $\mathrm{CD}^{+}, 6 \%$ in $\mathrm{CD}^{-}$) and Finegoldia (ASV761) (below $1 \%$ in $\mathrm{CD}^{+}, 2 \%$ in $\left.\mathrm{CD}^{-}\right)$compared to $\mathrm{CD}^{-}$cohort. In UC patients who later developed SkAE $\left(\mathrm{UC}^{+}\right)$, we observed higher

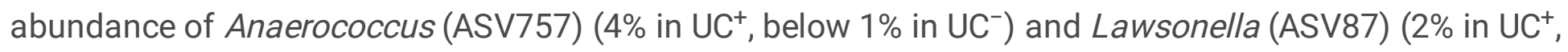

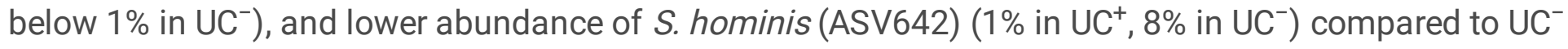
patients who did not develop SkAE. Interestingly, genus Corynebacterium (ASV86) was highly abundant in $\mathrm{UC}^{-}$cohort (17\%) and it was under the detection limit (set to $1 \%$ ) in $\mathrm{UC}^{+}$cohort. Healthy controls were shown to possess high levels of Cutibacterium (ASV212) (53\%) and Staphylococcus (ASV636) (15\%) and, to a lesser extent, also Corynebacterium (ASV86) (4\%). However, Corynebacterium (ASV86) in HC was present to a much lesser extent than in $\mathrm{UC}^{-}$patients who did not develop SkAE ( $4 \%$ in $\mathrm{HC}$ vs. $17 \%$ in $\mathrm{UC}^{-}$) (Supplementary Figure S3B). MetamicrobiomeR-based differential abundance analysis after multiple testing corrections revealed significant association of Gemella (ASV201), Enhydrobacter (ASV370), Pseudoclavibacter (ASV52), and Kocuria (ASV55) with $\mathrm{CD}^{+}$cohort (Supplementary Figure 
S3C). This analysis, however, showed no significant associations for UC cohort after multiple testing corrections.

\section{Incidence of SkAE during anti-TNFa therapy is associated with changes in the serum levels of biomarkers of epithelial barrier function}

To gain insight into the pathogenesis of skin side effects of anti-TNFa therapy, we examined different biomarkers related to skin and gut barrier function and to immune response. We analyzed 22 potential serum biomarkers at baseline, during the manifestation of SkAE, and after the healing of SkAE (Supplementary Figure S4). We found that a marker closely associated with epithelial integrity, L-FABP, was lowered during the manifestation of SkAE (Fig. 4A). Conversely, I-FABP increased significantly after SkAE were healed (Fig. 4A). Moreover, we showed that there were no differences in L-FABP, I-FABP, and EFABP at baseline between the groups of patients with and without SkAE (Supplementary Figure S5). Further correlation analysis of the clinical data together with potential biomarkers of SkAE identified several features associated with the occurrence of SkAE or specific to the absence of SkAE (Fig. 4B, Supplementary Figure S6). Interestingly, in patients suffering SkAE we found a positive correlation of (i) IFABP with TNFa levels $(r=0.906)$; (ii) BMI with the levels of TIMP-1 $(r=0.786)$, MMP-9 $(r=0.786)$, LBP $(r$ $=0.928)$, and EG-VGF ( $r=0.808)$; and (iii) osteoprotegerin with IBD severity $(r=0.866)$, calculated as Spearman correlation coefficient. Furthermore, SkAE affected patients showed a negative correlation of (i) fecal calprotectin with hemoglobin ( $r=-0.821)$, (ii) weight with osteoprotegerin $(r=0.786)$, and (iii) BMI with the levels of IGFII $(r=-0.786)$, which was not observed at baseline or at the study endpoint (Fig. 4B). In contrast, we found the same positive correlation pattern between the levels of FC and IBD severity at baseline and at the study endpoint, but not during SkAE incidence ( $r=0.954$ and $r=0.845)$ (Fig. 4B). There was no association of SkAE to blood levels of anti-TNFa (data not shown).

\section{Discussion}

The lack of tools to predict the development of skin adverse events (SkAE) after therapy with TNFa blockers in IBD patients [34,35] prompted us to investigate skin microbiota composition and serum biomarkers pattern in these patients.

We separately analyzed several skin localities, i.e., the retroauricular crease, the lumbar region, and the inguinal crease, to avoid inconsistency in results caused by sampling and comparing different skin ecological niches $[29,36]$. The results of our study demonstrate that CD patients have distinct skin microbiota signature distinguishing them from UC and HC. Although differences in gut microbiota composition between $\mathrm{CD}, \mathrm{UC}$, and $\mathrm{HC}$ were reported [37-39], the changes in skin microbiota composition we observed are rather new and unexplored. For the first time, we show that the richness and evenness represented here by Shannon entropy of skin bacterial communities at the retroauricular crease was higher in $\mathrm{CD}$ patients than in UC patients and $\mathrm{HC}$, and that this phenomenon did not depend on the treatment (neither anti-TNFa, nor anti-IL12/23), sex, or BMI. The number of observed ASVs and Chao1 index were without statistical differences in $\mathrm{CD}, \mathrm{UC}$, and $\mathrm{HC}$. In addition, the lumbar region but not the 
inguinal crease showed significant differences between $\mathrm{CD}$ and $\mathrm{HC}$ in all three alpha diversity metrics. Although not significantly so, we observed lower Shannon entropy in CD compared to UC and HC only at the inguinal crease. This can only reflect the respective ecological niches' characteristics [40], but further studies will be needed to confirm or refute whether these changes relate also to the particular form of the disease.

While IBD patients have generally decreased microbiota diversity in the gut [22,38], here we show that their skin microbiota diversity is increased compared to $\mathrm{HC}$, particularly at the retroauricular crease and in the lumbar region. Importantly, the increased diversity was not due to treatment since it was observed already at baseline and it was maintained throughout the whole study. We have previously reported a similar tendency towards increased skin microbiota diversity in psoriatic skin lesions and unaffected psoriatic skin [29], while the intestinal microbiota showed the opposite trend when compared to healthy controls [41]. Similarly, rosacea patients tended to have an increased abundance of various types of bacterial strains on the skin [42], while their gut microbiota richness was reduced [43]. Even cirrhosis was recently shown to be associated with alterations in the skin microbiota composition [44].

The inguinal crease is a moist skin environment, so according to Grice et al. (2009), its diversity should exceed that of the sebaceous sites [36]. This was true for UC and HC but not for CD patients, where the inguinal crease showed lower diversity than the lumbar region. From a general point of view, the skin microbiota composition of UC patients was more similar to that of HC than CD patients. Here we show that $\mathrm{CD}$ has a much stronger impact on the skin microbiota than UC and that each disease might affect a different microbial niche. Our finding of skin microbiota similarity between $\mathrm{UC}$ and $\mathrm{HC}$ is in line with a study focused on gut microbiota in IBD patients, where the authors observed that the difference in beta diversity between $\mathrm{HC}$ and $\mathrm{UC}$ was smaller than between $\mathrm{HC}$ and $\mathrm{CD}$, indicating more overlapping species between $\mathrm{HC}$ and UC group [45]. Despite the shared species between UC and HC, gut microbiota samples of IBD patients clustered by diagnosis and had a lower alpha diversity compared to healthy controls [12, $22,38,46]$.

The general composition of the main bacterial phyla of IBD patients at the retroauricular crease did not markedly differ from the previously published skin microbiota of $\mathrm{HC}$ [36, 47-49], as it was dominated by Actinobacteria and Firmicutes and to a lesser extent by Proteobacteria. The most abundant bacterial genera in all samples were Cutibacterium (ASV2012), Staphylococcus (ASV636), and Corynebacterium (ASV86), followed by the species Staphylococcus hominis (ASV642) and Staphylococcus epidermidis (ASV640). Cutibacteria, corynebacteria, and staphylococci are abundant colonizers of the skin, but under certain conditions can frequently cause opportunistic infections [50,51]. Differential abundance analysis (DAA) of the microbiota associated with the retroauricular crease showed that compared to HC, Corynebacterium (ASV86) was significantly associated with CD, while Delftia (ASV1039) was associated with UC patients. Skin microbiota profile of both CD and UC was linked with higher abundance of Pseudomonas (ASV1183). 
Although Delftia is rarely a clinically significant species, it is able to cause infections in both immunocompetent and immunocompromised individuals [52]. Similarly, pseudomonas infections usually develop in immunocompromised individuals, but healthy people might also be sensitive to this skin commensal [53]. This highlights the need to consider that the same microbe can play diverse roles in different settings. Microbial behavior can change depending on the physiological state or health of the host, for example, microbes can switch metabolite production in response to certain conditions [54-56]. Thus, the pro-inflammatory IBD environment might impact the gut-skin axis, promoting shifts in the abundance of specific microbial species on the skin. In this way, patients might be more/less susceptible either to IBD-related skin manifestations or to skin adverse events (SkAE) often associated with anti-TNFa therapy.

We hypothesized that IBD patients might have a specific skin microbiota composition that could predispose them to develop SkAE after anti-TNFa treatment. These paradoxical reactions may be induced by the feedback response to the blockade of TNFa, which selectively maintains type I interferon production by plasmacytoid dendritic cells [57]. By examining patients before the initiation of anti-TNFa treatment, we found that certain skin microbes were associated with individuals that would later go on to develop SkAE. The relative frequency of the most abundant taxa showed that skin swab samples from CD patients who later developed SkAE $\left(\mathrm{CD}^{+}\right)$were predominated by Staphylococcus (ASV636) $(29 \%$ in $C D^{+}$vs. $13 \%$ in $\mathrm{CD}^{-}$). Furthermore, the $\mathrm{CD}^{+}$cohort showed a higher abundance of Staphylococcus aureus (ASV638) than the $\mathrm{CD}^{-}$cohort ( $1 \%$ in $\mathrm{CD}^{+}$vs. below $1 \%$ in $\mathrm{CD}^{-}$). The presence of $S$. aureus could be also a risk factor for atopic dermatitis development [58]. Moreover, the relative abundance of staphylococci, particularly S. aureus and S. epidermidis increased in atopic dermatitis flares [59]. In agreement with Byrd et al. (2017), we observed not only an increase of $S$. aureus, but also an increase of $S$. epidermidis in $C^{+}$ cohort which are patients prone to develop SkAE after anti-TNFa treatment $\left(10 \%\right.$ in $\mathrm{CD}^{+}$vs. $5 \%$ in $\left.\mathrm{CD}^{-}\right)$.

The genus Dietzia, sometimes misidentified as Rhodococcus spp. [60], is considered an opportunistic pathogen, often associated with various infections [61]. We observed its elevated abundance in $\mathrm{CD}^{+}$over $\mathrm{CD}^{-}$cohort ( $4 \%$ vs. below $1 \%$, respectively), which is in concordance with another study exploring skin microbiota in atopic dermatitis [62]. Similarly, we showed association of Enhydrobacter with skin of CD patients prone to develop SkAE and decreased abundance of Corynebacterium in these patients. Furthermore, Pseudoclavibacter, significantly associated with $\mathrm{CD}^{+}$patients, was shown to be enriched in S. aureus-high skin psoriasis samples [63]. Apart from Enhydrobacter and Pseudoclavibacter, our metamicrobiomeR analysis revealed also Gemella to be significantly associated with $\mathrm{CD}^{+}$skin. Likewise, another study confirmed enrichment of Gemella in atopic dermatitis-prone individuals [64]. This proposes an undeniable role of these microbes in different skin conditions. Based on our results, we suggest that skin microbes can interfere with the surrounding microbiota and may predispose IBD patients for SkAE manifestation during anti-TNFa therapy.

Anaerococcus belongs to the group of Gram-positive anaerobic cocci (GPAC). While GPAC constitute a major part of the commensal microbiota of the skin and mucosal surfaces [65], they are frequently 
involved in opportunistic infections in immunocompromised or otherwise vulnerable individuals $[66,67]$. We observed that skin microbiota in UC patients who later developed SkAE $\left(\mathrm{UC}^{+}\right)$showed high abundance of Anaerococcus species (ASV757) (4\%) compared to UC', where its abundance was below $1 \%$. In contrast, Anaerococcus (ASV757) levels in $\mathrm{CD}^{-}$patients reached $6 \%$ and they were below detection limit (below $1 \%$ ) in $\mathrm{CD}^{+}$patients. This suggests that microbes of one species can facilitate/protect from the manifestation of SkAE in a context-dependent manner. Although immensely interesting, these results are difficult to interpret as the analysis of predisposition to SkAE by differential abundance analysis did not provide statistically significant results due to the low number of UC + patients, where potential differences might be masked by normal interpersonal variation. The microbiota of $C D$ and UC patients is likely to have different settings by the very nature of the disease, and hence exerts different effects on host physiology, including, for example, the ability to respond to treatment, as well as the character of the response itself [68]. Although this theory is widely accepted, the power of our study is necessarily limited by the moderate number of patients with SkAE overall and the diverse forms these SkAE took.

Dysbiosis and gut-skin axis participation are common features of many inflammatory diseases like IBD or psoriasis, in the pathogenesis of which TNFa plays an important role $[69,70]$. Psoriasis is a common comorbidity of IBD, with a prevalence of $3.6 \%$ in $C D$ and $2.8 \%$ in UC [71], and patients with psoriasis have a 2.5-fold higher risk of developing $C D$ and a 1.7-fold higher risk of developing UC than the general population [72]. In both IBD and psoriasis, multiple common pathogenic mechanisms have been proposed, including increased intestinal permeability $[29,73]$ and an aberrant immune response to the microbiota [74]. In pediatric UC, microbial changes were observed at the sites of inflammation and mucin production was lowered even in non-inflamed parts of the bowel. A thinned mucous layer impairs the barrier function and may allow bacterial penetration, promoting the release of inflammatory markers [79]. This suggests that changes of microbiota composition and function might directly or indirectly impact the skin barrier role. In addition, Genome Wide Association Studies (GWAS) uncovered that IBD and psoriasis share 4 susceptibility loci, which contain several shared genes involved in innate and adaptive immunity [75]. The link between the two diseases has been further strengthened by the finding that they can appear to each other as paradoxical treatment-related adverse events [76-78]. As mentioned earlier, IBD patients develop multiple types of skin manifestations and patients receiving anti-TNFa therapy often suffer from skin adverse events. Due to the lack of scientific evidence, however, it is not known whether TNFa inhibitors affect the composition of skin microbiota. We did not observe any effect of this treatment neither on the skin nor on the gut microbiota composition [38] and other studies report only modest differences in the gut microbiota composition after anti-TNFa therapy [80]. Magnusson et al. (2016), on the other hand, showed that treatment with adalimumab improved the symptoms of UC and restored the gut microbiota of UC patients. Specifically, the authors have described an increase in the abundance of Faecalibacterium prausnitzii and a decrease in disease severity in responders after antiTNFa treatment [81]. However, changes in gut microbiota after anti-TNFa therapy do not have to be necessarily caused directly by anti-TNFa treatment. Reversal of gut dysbiosis may simply go hand in hand with the improvement of the disease symptoms [82]. Together, these findings imply a dynamic interplay between the skin and gut homeostasis and highlight the important role of many gut-skin axis

Page $14 / 26$ 
mediators, such as markers of inflammation or epithelial barrier function. These markers might reflect disease activity and are potentially useful for early diagnosis and prognosis.

To confirm the gut-skin axis involvement in SkAE manifestation, we searched for biomarkers related to the disruption of epithelial barrier integrity in our study cohort of IBD patients. One of the selected biomarkers, I-FABP, has been previously described to be elevated in patients with psoriasis compared to healthy controls $[29,83]$. However, this finding is not in line with our current results. We found a decreased production of I-FABP and L-FABP during the onset of SkAE and a subsequent increase after SkAE healing. Moreover, overexpression of E-FABP by keratinocytes has been described in patients with psoriasis [84, 85], but was not observed during SkAE manifestation in this study. There are two possible explanations for these unexpected results. First, the patients in this study experienced different types of SkAE, and the role of I-FABP, L-FABP, and E-FABP in the pathogenesis of drug exanthema or eczema might differ from that in psoriasis. Second, during different time points such as (i) baseline, (ii) the manifestation of SkAE, (iii) and the study endpoint, different mechanism of inflammatory control might prevail. We showed a positive correlation of I-FABP with TNFa levels during the occurrence of SkAE, indicating increased production of epithelial barrier disruption markers during inflammation. Osteoprotegerin (OPG) levels correlated positively with disease severity during the onset of SkAE. This finding is in agreement with previous studies that found an increased OPG in IBD patients [15, 86]. Our results highlight the hitherto unexplained relationships between the gut-skin axis serum biomarkers, anti-TNFa therapy, and the development of SkAE in IBD patients. Our findings encourage further investigation of the inflammatory mechanisms in SkAE manifestation.

\section{Conclusions}

We have shown a specific skin microbiota signature that distinguishes CD patients from UC patients and $\mathrm{HC}$, suggesting that the changes in microbiota composition in IBD are not limited to the gut. This so far unique observation is another proof of the gut-skin axis interconnection in IBD, and together with other follow-up studies will pose new directions for investigation in this field. In addition, the presence or absence of certain skin microbes might predispose IBD patients for SkAE manifestation, although further investigation is necessary. The levels of serum markers of impaired epithelial barrier integrity may reflect the involvement of different inflammatory mechanisms during SkAE manifestation. Our results provide an important basis for future studies, the results of which could then serve as a prognostic tool to predict the manifestation of SkAE prior to anti-TNFa treatment.

\section{Declarations}

\section{Ethics approval and consent to participate:}

The study was conducted according to the guidelines of the Declaration of Helsinki. This study was approved by the Ethics Committee of ISCARE (Nr2017/Ila), IKEM (G 17-06-09), and University Hospital Bulovka (21.6.2017 I8513IEK-Z). 


\section{Consent for publication:}

Not applicable.

\section{Availability of data and material:}

Data from amplicon sequencing are available in the Sequence Read Archive (SRA) under the accession number SUB10259106 and BioProject ID PRJNA757573. Part of the data will be available on request.

\section{Competing interests:}

The authors declare that they have no competing interests.

\section{Funding:}

This research was funded by the Ministry of Health of the Czech Republic, grant number NV18-09-00493, and Institutional Research Concept (RVO: 61388971).

\section{Author's contributions:}

ZJZ, HT-H, MK, ML, FR and JH conceived and design the research. FR, MK, LB, MN, MV, and ML examined the patients and healthy controls and collected samples. DS and JK performed the analysis of the sequencing data and statistics. ZS proceeded the skin swab samples and analyzed and interpreted the sequencing data. ZS and ZJZ wrote the manuscript. ZJ, RR, SC, MM, and TT analyzed the biomarkers in patient's sera and together with ZJZ, KK, and NG interpreted the data. All authors revised and approved the final version of the manuscript.

\section{Acknowledgments:}

We would like to thank Alena Kubatova for excellent technical support and to all study nurses from ISCARE and Bulovka Hospital. We acknowledge the CF Genomics of CEITEC supported by the NCMG research infrastructure (LM2015091 funded by MEYS CR) for their support with obtaining scientific data presented in this paper.

\section{References}

1. Alatab S. et al., The global, regional, and national burden of inflammatory bowel disease in 195 countries and territories, 1990-2017: a systematic analysis for the Global Burden of Disease Study 2017. The Lancet gastroenterology \& hepatology 5, 17-30 (2020).

2. Jarkovský J. et al., Epidemiologie, hospitalizační léčba a migrace IBD pacientů za specializovanou péčí v České republice. Gastroenterol Hepatol 71, 501-509 (2017).

3. K Ko J. et al., Inflammatory bowel disease: etiology, pathogenesis and current therapy. Current pharmaceutical design 20, 1082-1096 (2014). 
4. Vavricka S.R. et al., Frequency and risk factors for extraintestinal manifestations in the Swiss inflammatory bowel disease cohort. Official journal of the American College of Gastroenterologyl ACG 106, 110-119 (2011).

5. Georgiou S. et al., Cutaneous manifestations of inflammatory bowel disease. Hospital chronicles 1, 158-168 (2006).

6. Huang B.L. et al., Skin manifestations of inflammatory bowel disease. Frontiers in physiology 3,13 (2012).

7. Kornbluth A., Infliximab approved for use in Crohn's disease: a report on the FDA GI Advisory Committee conference. Inflammatory bowel diseases 4, 328-329 (1998).

8. Cleynen I. et al., Paradoxical inflammation induced by anti-TNF agents in patients with IBD. Nature reviews Gastroenterology \& hepatology 9, 496-503 (2012).

9. Fidder H. et al., Long-term safety of infliximab for the treatment of inflammatory bowel disease: a single-centre cohort study. Gut 58, 501-508 (2009).

10. Mocci G. et al., Dermatological adverse reactions during anti-TNF treatments: focus on inflammatory bowel disease. Journal of Crohn's and Colitis 7, 769-779 (2013).

11. Nigam G.B. et al., Systematic review and meta-analysis of dermatological reactions in patients with inflammatory bowel disease treated with anti-tumour necrosis factor therapy. European Journal of Gastroenterology \& Hepatology 33, 346-357 (2021).

12. Bajer L. et al., Distinct gut microbiota profiles in patients with primary sclerosing cholangitis and ulcerative colitis. World journal of gastroenterology 23, 4548 (2017).

13. Pittayanon R. et al., Differences in gut microbiota in patients with vs without inflammatory bowel diseases: a systematic review. Gastroenterology 158, 930-946. e931 (2020).

14. Caruso R. et al., Host-microbiota interactions in inflammatory bowel disease. Nature Reviews Immunology 20, 411-426 (2020).

15. Coufal S. et al., Inflammatory bowel disease types differ in markers of inflammation, gut barrier and in specific anti-bacterial response. Cells 8, 719 (2019).

16. Clark C. et al., in JOURNAL OF CROHNS \& COLITIS. (OXFORD UNIV PRESS GREAT CLARENDON ST, OXFORD OX2 6DP, ENGLAND, 2016), vol. 10, pp. S129-S129.

17. Pujada A. et al., Matrix metalloproteinase MMP9 maintains epithelial barrier function and preserves mucosal lining in colitis associated cancer. Oncotarget 8, 94650 (2017).

18. Schumann R.R. et al., A novel acute-phase marker: lipopolysaccharide binding protein (LBP). (1999).

19. Winter J. et al., Human defensins: potential tools for clinical applications. Polymers 4, 691-709 (2012).

20. Hold G.L. et al., Role of the gut microbiota in inflammatory bowel disease pathogenesis: what have we learnt in the past 10 years? World journal of gastroenterology: WJG 20, 1192 (2014).

21. Packey C. et al., Interplay of commensal and pathogenic bacteria, genetic mutations, and immunoregulatory defects in the pathogenesis of inflammatory bowel diseases. Journal of internal 
medicine 263, 597-606 (2008).

22. Sokol H. et al., Fungal microbiota dysbiosis in IBD. Gut 66, 1039-1048 (2017).

23. Tlaskalová-Hogenová $\mathrm{H}$. et al., The role of gut microbiota (commensal bacteria) and the mucosal barrier in the pathogenesis of inflammatory and autoimmune diseases and cancer: contribution of germ-free and gnotobiotic animal models of human diseases. Cellular \& molecular immunology $\mathbf{8}$, 110-120 (2011).

24. Harbord M. et al., The first European evidence-based consensus on extra-intestinal manifestations in inflammatory bowel disease. Journal of Crohn's and Colitis 10, 239-254 (2016).

25. Maaser C. et al., ECCO-ESGAR Guideline for Diagnostic Assessment in IBD Part 1: Initial diagnosis, monitoring of known IBD, detection of complications. Journal of Crohn's and Colitis 13, 144-164K (2019).

26. Harvey R. et al., A simple index of Crohn's-disease activity. The Lancet 315, 514 (1980).

27. Schroeder K.W. et al., Coated oral 5-aminosalicylic acid therapy for mildly to moderately active ulcerative colitis. New England Journal of Medicine 317, 1625-1629 (1987).

28. Consortium H.M.P., Structure, function and diversity of the healthy human microbiome. nature 486, 207 (2012).

29. Stehlikova Z. et al., Dysbiosis of skin microbiota in psoriatic patients: co-occurrence of fungal and bacterial communities. Frontiers in microbiology 10, 438 (2019).

30. Stehlikova Z. et al., Oral microbiota composition and antimicrobial antibody response in patients with recurrent aphthous stomatitis. Microorganisms 7, 636 (2019).

31. Stehlikova Z. et al., Crucial role of microbiota in experimental psoriasis revealed by a gnotobiotic mouse model. Frontiers in microbiology 10, 236 (2019).

32. Bates D. et al., Fitting linear mixed-effects models using Ime4. arXiv preprint arXiv:1406.5823, (2014).

33. Ho N.T. et al., metamicrobiomeR: an R package for analysis of microbiome relative abundance data using zero-inflated beta GAMLSS and meta-analysis across studies using random effects models. BMC bioinformatics 20, 1-15 (2019).

34. Cossio M.-L. et al., Skin manifestations in pediatric patients treated with a TNF-alpha inhibitor for inflammatory bowel disease: a retrospective study. Journal of Cutaneous Medicine and Surgery 24, 333-339 (2020).

35. Sridhar S. et al., Dermatological manifestations in pediatric patients with inflammatory bowel diseases on anti-TNF therapy. Inflammatory bowel diseases 24, 2086-2092 (2018).

36. Grice E.A. et al., Topographical and temporal diversity of the human skin microbiome. science 324 , 1190-1192 (2009).

37. Andoh A. et al., Comparison of the fecal microbiota profiles between ulcerative colitis and Crohn's disease using terminal restriction fragment length polymorphism analysis. Journal of gastroenterology 46, 479-486 (2011). 
38. Schierova D. et al., Fecal Microbiome Changes and Specific Anti-Bacterial Response in Patients with IBD during Anti-TNF Therapy. Cells 10, 3188 (2021).

39. Swidsinski A. et al., Active Crohn's disease and ulcerative colitis can be specifically diagnosed and monitored based on the biostructure of the fecal flora. Inflammatory bowel diseases 14, 147-161 (2008).

40. Grice E.A. et al., Topographical and temporal diversity of the human skin microbiome. science 324, 1190-1192 (2009).

41. Shapiro J. et al., Psoriatic patients have a distinct structural and functional fecal microbiota compared with controls. The Journal of dermatology 46, 595-603 (2019).

42. Wang R. et al., Bacterial and fungal microbiome characterization in patients with rosacea and healthy controls. The British Journal of Dermatology, (2020).

43. Chen Y.-J. et al., An altered fecal microbial profiling in rosacea patients compared to matched controls. Journal of the Formosan Medical Association 120, 256-264 (2021).

44. Bajaj J.S. et al., Alterations in skin microbiomes of patients with cirrhosis. Clinical Gastroenterology and Hepatology 17, 2581-2591. e2515 (2019).

45. Jagadesan Sankarasubramanian R.A. et al., Gut Microbiota and Metabolic Specificity in Ulcerative Colitis and Crohn's Disease. Frontiers in Medicine 7, (2020).

46. Sankarasubramanian J. et al., Gut microbiota and metabolic specificity in ulcerative colitis and Crohn's disease. Frontiers in medicine, 874 (2020).

47. Costello E.K. et al., Bacterial community variation in human body habitats across space and time. science 326, 1694-1697 (2009).

48. Grice E.A. et al., The skin microbiome. Nature reviews microbiology 9, 244-253 (2011).

49. Oh J. et al., Biogeography and individuality shape function in the human skin metagenome. Nature 514, 59-64 (2014).

50. Otto M., Staphylococcus colonization of the skin and antimicrobial peptides. Expert review of dermatology 5, 183-195 (2010).

51. et al. (s Note: MDPI stays neutral with regard to jurisdictional claims in published ...

52. Bilgin H. et al., Delftia acidovorans: a rare pathogen in immunocompetent and immunocompromised patients. Canadian Journal of Infectious Diseases and Medical Microbiology 26, 277-279 (2015).

53. Wu D.C. et al., Pseudomonas skin infection. American journal of clinical dermatology 12, 157-169 (2011).

54. Otto M., Staphylococcus epidermidis-the'accidental'pathogen. Nature reviews microbiology 7, 555567 (2009).

55. Ryu S. et al., Colonization and infection of the skin by S. aureus: immune system evasion and the response to cationic antimicrobial peptides. International journal of molecular sciences $15,8753-$ 8772 (2014). 
56. Takahashi T. et al., The critical and multifunctional roles of antimicrobial peptides in dermatology. Dermatologic clinics 35, 39-50 (2017).

57. Conrad C. et al., TNF blockade induces a dysregulated type I interferon response without autoimmunity in paradoxical psoriasis. Nature communications 9, 1-11 (2018).

58. Kong H.H. et al., Temporal shifts in the skin microbiome associated with disease flares and treatment in children with atopic dermatitis. Genome research 22, 850-859 (2012).

59. Byrd A.L. et al., Staphylococcus aureus and Staphylococcus epidermidis strain diversity underlying pediatric atopic dermatitis. Science translational medicine 9, eaal4651 (2017).

60. Koerner R.J. et al., The genus Dietzia: a new home for some known and emerging opportunist pathogens. FEMS Immunology \& Medical Microbiology 55, 296-305 (2009).

61. Navaratnam J. et al., Identification of Dietzia species in a patient with endophthalmitis following penetrating injury with retained intraocular metallic foreign body. Case reports in infectious diseases 2018, (2018).

62. Noll M. et al., Improvement of atopic dermatitis by synbiotic baths. Microorganisms 9, 527 (2021).

63. Chang H.-W. et al., Alteration of the cutaneous microbiome in psoriasis and potential role in Th17 polarization. Microbiome 6, 1-27 (2018).

64. Chng K.R. et al., Whole metagenome profiling reveals skin microbiome-dependent susceptibility to atopic dermatitis flare. Nature microbiology 1, 1-10 (2016).

65. Murdoch D., Gram-positive anaerobic cocci. Clinical microbiology reviews 11, 81-120 (1998).

66. Wall I.B. et al., Potential role of anaerobic cocci in impaired human wound healing. Wound Repair and Regeneration 10, 346-353 (2002).

67. Wildeboer-Veloo A. et al., Development of 16S rRNA-based probes for the identification of Grampositive anaerobic cocci isolated from human clinical specimens. Clinical microbiology and infection 13, 985-992 (2007).

68. Vasudevan A. et al., Time to clinical response and remission for therapeutics in inflammatory bowel diseases: What should the clinician expect, what should patients be told? World journal of gastroenterology 23, 6385 (2017).

69. Ruder B. et al., Tumour necrosis factor alpha in intestinal homeostasis and gut related diseases. International journal of molecular sciences 20, 1887 (2019).

70. Yost J. et al., The role of TNF inhibitors in psoriasis therapy: new implications for associated comorbidities. F1000 medicine reports 1, (2009).

71. Alinaghi F. et al., Global prevalence and bidirectional association between psoriasis and inflammatory bowel disease-a systematic review and meta-analysis. Journal of Crohn's and Colitis $14,351-360$ (2020).

72. Fu Y. et al., Association of psoriasis with inflammatory bowel disease: a systematic review and metaanalysis. JAMA dermatology 154, 1417-1423 (2018). 
73. Chang J. et al., Impaired intestinal permeability contributes to ongoing bowel symptoms in patients with inflammatory bowel disease and mucosal healing. Gastroenterology 153, 723-731. e721 (2017).

74. Bouma G. et al., The immunological and genetic basis of inflammatory bowel disease. Nature Reviews Immunology 3, 521-533 (2003).

75. Skroza N. et al., Correlations between psoriasis and inflammatory bowel diseases. BioMed research international 2013, (2013).

76. Lolli E. et al., Psoriasis phenotype in inflammatory bowel disease: a case-control prospective study. Journal of Crohn's and Colitis 9, 699-707 (2015).

77. Nehring P. et al., Is psoriasis treatment a risk factor for inflammatory bowel disease? Pharmaceutical Medicine 34, 257-262 (2020).

78. Vlachos C. et al., Psoriasis and inflammatory bowel disease: links and risks. Psoriasis (Auckland, NZ) 6, 73 (2016).

79. Alipour M. et al., Mucosal barrier depletion and loss of bacterial diversity are primary abnormalities in paediatric ulcerative colitis. Journal of Crohn's and Colitis 10, 462-471 (2016).

80. Bazin T. et al., Microbiota composition may predict anti-TNF alpha response in spondyloarthritis patients: an exploratory study. Scientific reports 8, 1-11 (2018).

81. Magnusson M.K. et al., Anti-TNF therapy response in patients with ulcerative colitis is associated with colonic antimicrobial peptide expression and microbiota composition. Journal of Crohn's and Colitis 10, 943-952 (2016).

82. Aldars-García L. et al., Systematic Review: The Gut Microbiome and Its Potential Clinical Application in Inflammatory Bowel Disease. Microorganisms 9, 977 (2021).

83. Sikora M. et al., Intestinal fatty acid binding protein, a biomarker of intestinal barrier, is associated with severity of psoriasis. Journal of clinical medicine 8, 1021 (2019).

84. Owczarczyk-Saczonek A. et al., Effect of methotrexate treatment on the expression of epidermal-fatty acid-binding protein (E-FABP) and apolipoproteins in patients with psoriasis. Advances in Dermatology and Allergology/Post py Dermatologii i Alergologii 37, 401 (2020).

85. Watarai A. et al., Nestin expression is increased in the suprabasal epidermal layer in psoriasis vulgaris. Acta dermato-venereologica 93, 39-43 (2013).

86. Moschen A. et al., The RANKL/OPG system is activated in inflammatory bowel disease and relates to the state of bone loss. Gut 54, 479-487 (2005).

\section{Figures}


Figure 1

$\mathbf{A}$
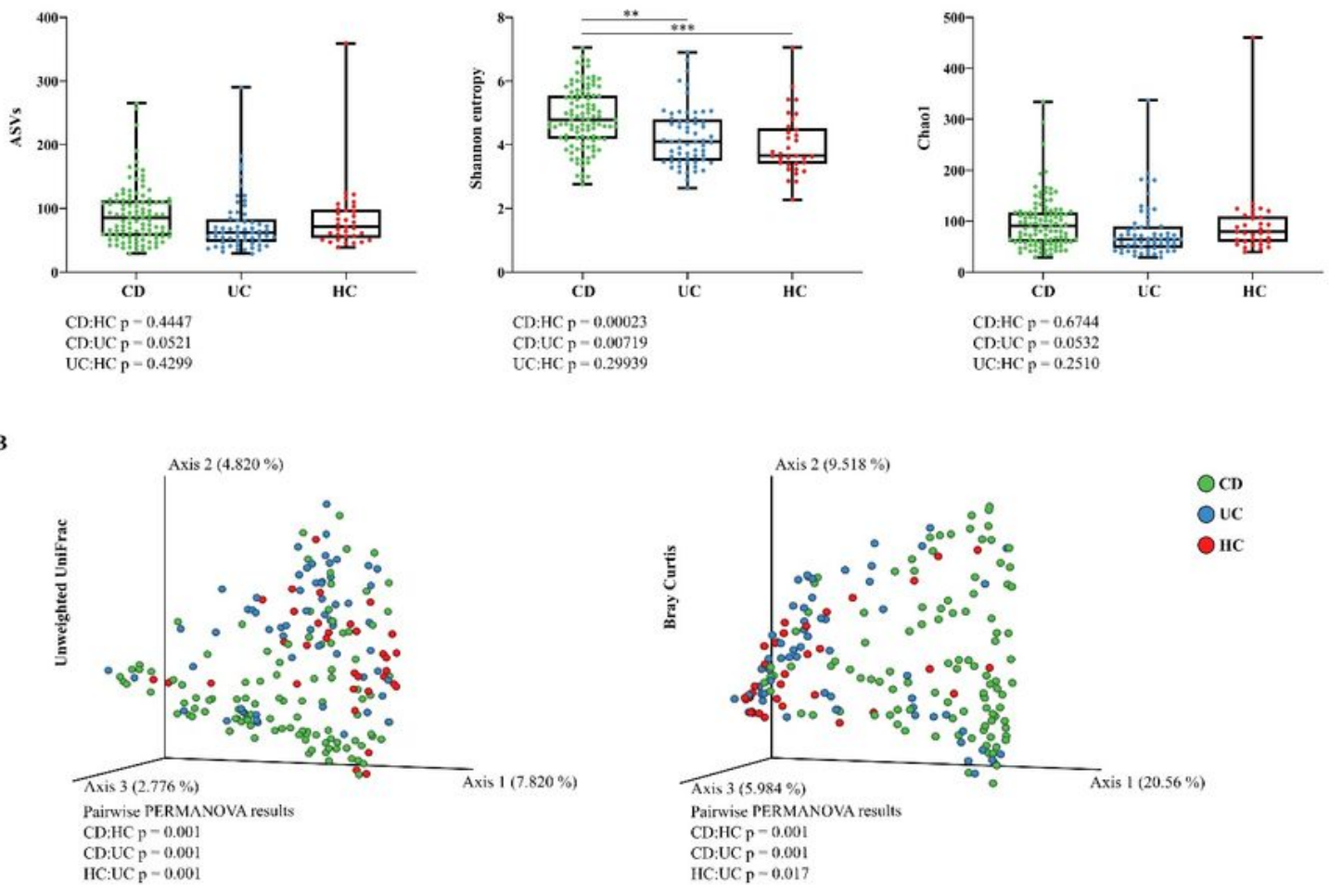

C
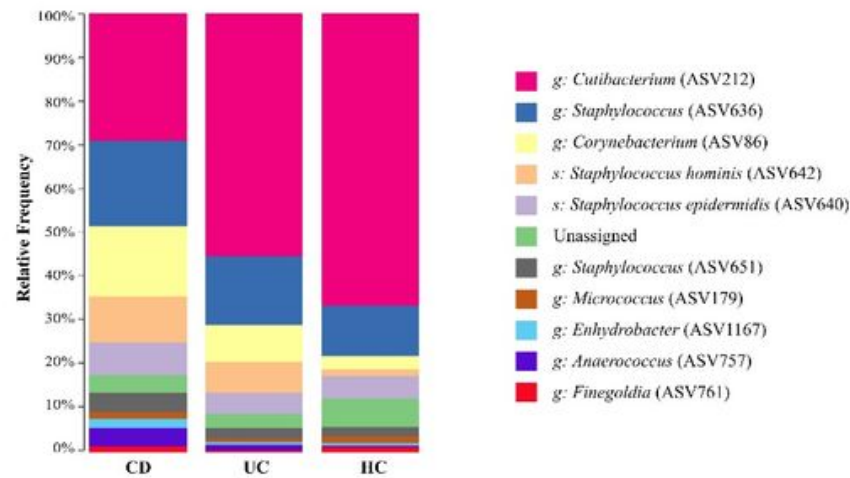

Skin microbiota diversity differs between CD, UC, and $\mathrm{HC}$ at the retroauricular crease. (A) Comparison of alpha diversity indices between $\mathrm{CD}, \mathrm{UC}$, and $\mathrm{HC}$. Each point represents one sample. The differences were analyzed by Linear mixed effect models (LMM) and subsequent Tukey post hoc test. (B) Principal coordinate analysis of the Bray-Curtis distance between $\mathrm{CD}, \mathrm{UC}$, and HC. Each point represents one sample. Groups were compared by PERMANOVA. (C) Relative abundance of the top 10 most abundant taxa of skin microbiota at the genus and species level. In all panels: ${ }^{*} \mathrm{p}<0.05 ;{ }^{*} \mathrm{p}<0.01 ;{ }^{* *} \mathrm{p}<0.001$. ASVs, amplicon sequence variants; $\mathrm{CD}$, Crohn's disease; UC, ulcerative colitis; $\mathrm{HC}$, healthy controls.

\section{Figure 1}

See image above for figure legend. 


\section{Figure 2}

A

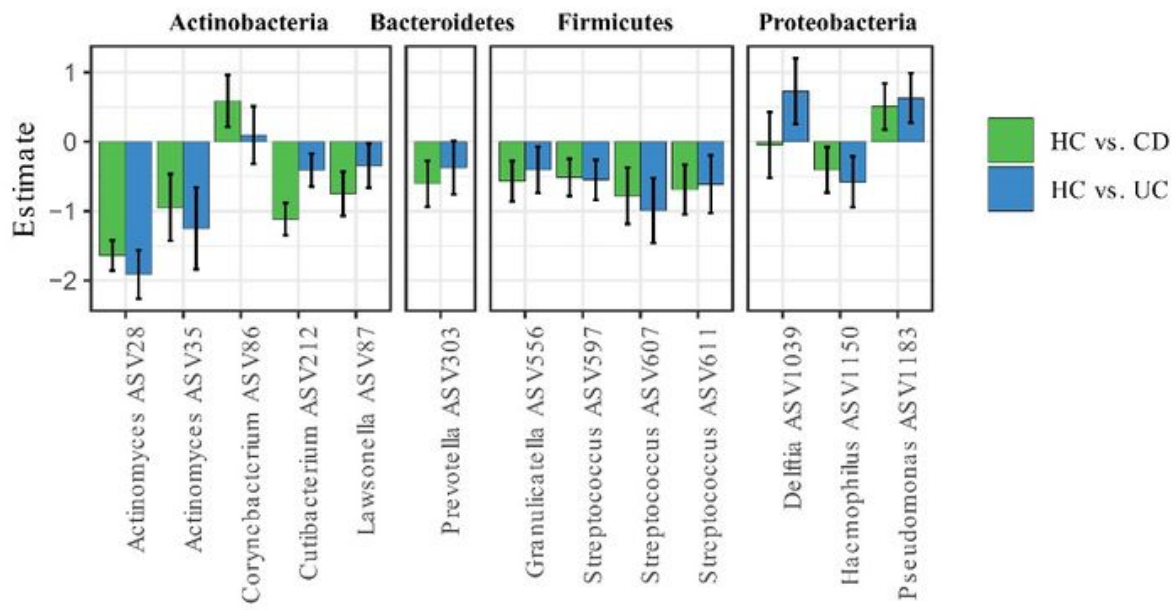

B

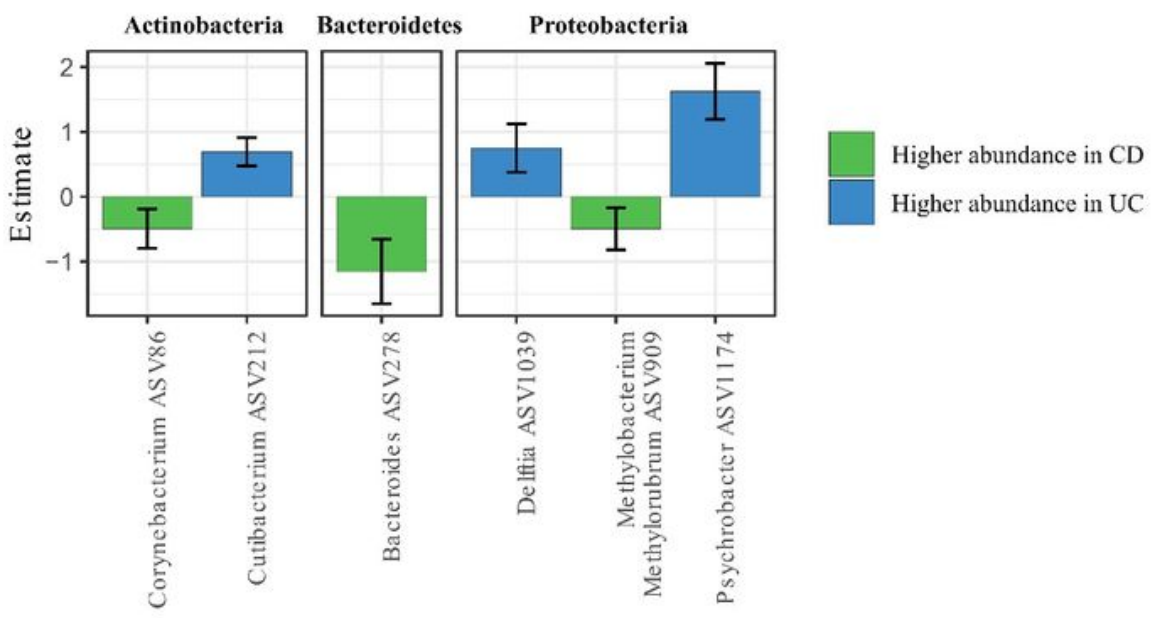

Specific microbiota pattern at the retroauricular crease distinguishes CD, UC and healthy controls. (A) Differential abundance analysis (DAA) of ASVs between CD and UC versus HC. Model estimates and 95\% confidence intervals are shown. Positive and negative values indicate an increase and decrease in abundance, respectively, in the IBD groups compared with $\mathrm{HC}$. Green color indicates ASVs abundance changes in CD compared to $\mathrm{HC}$ and blue color abundance changes between UC and HC. (B) Significant differences in taxa between CD and UC assessed by Differential abundance analysis. The taxa with significantly higher abundances in CD over UC patients are shown as negative estimates (color-coded in green), and the taxa with significantly higher abundances in UC over CD patients are shown as positive estimates (color-coded in blue). ASVs with relative abundance of more than $0.01 \%$ in all samples and detected in at least $10 \%$ samples were used in DAA analysis. CD, Crohn's disease; UC, ulcerative colitis; $\mathrm{HC}$, healthy controls.

\section{Figure 2}

See image above for figure legend. 


\section{Figure 3}

A
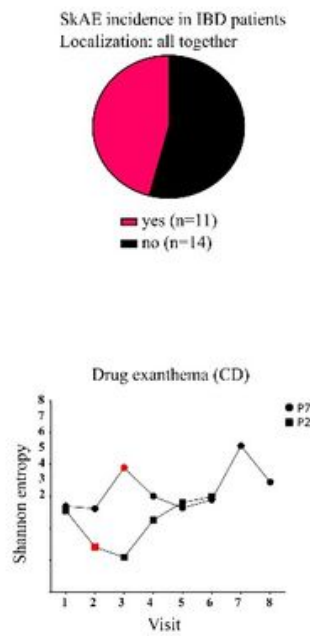

C

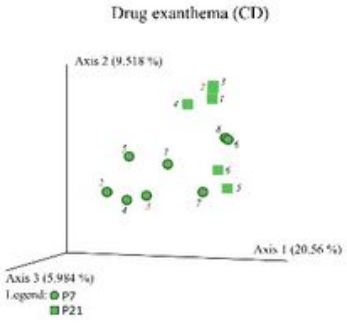

B

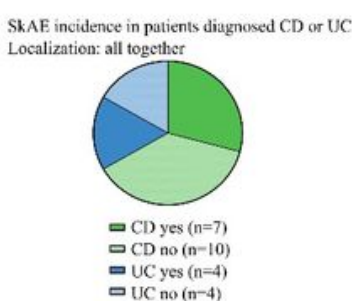

SkAl: manifestation in patients diagnosed CD or UC

Locealization: Retroauricular crease

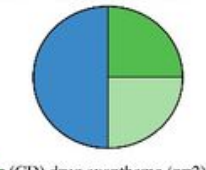

( $(\mathrm{CD})$ drug exanthema $(\mathrm{n}=2)$

(CD) eczema $(\mathrm{n}-2)$

a (UC) papulopustular exanthema $(\mathrm{n}=4)$
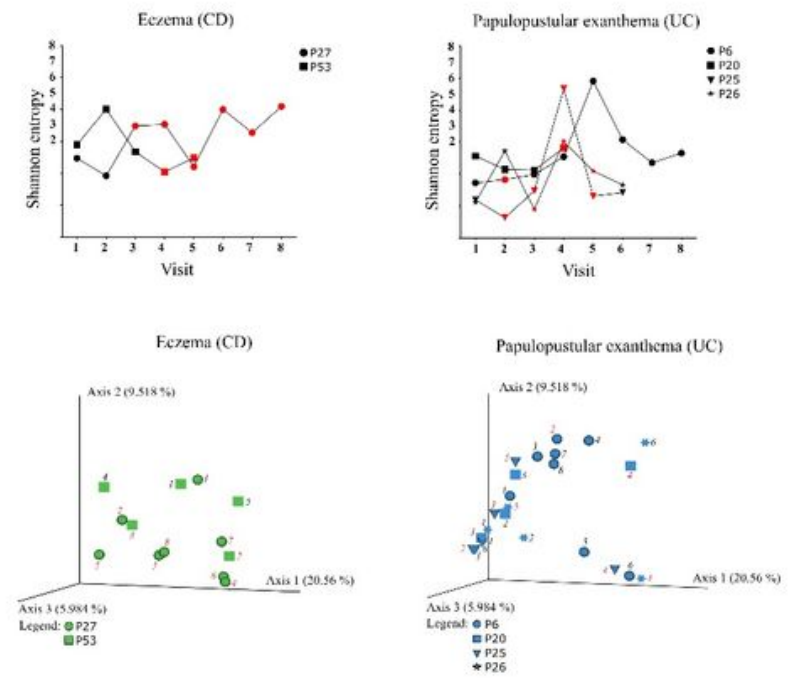

D
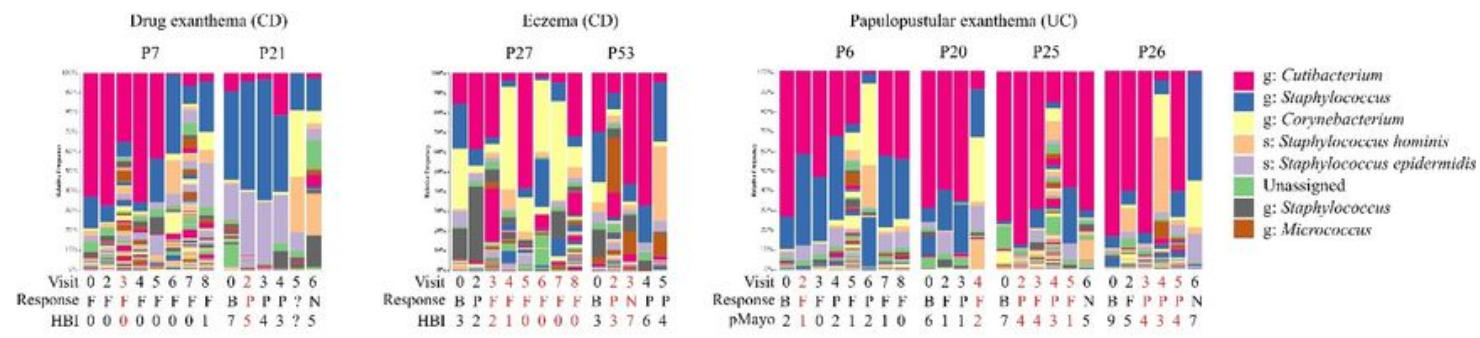

Characteristics of the IBD patients suffering from SkAE and their microbiota of retroauricular crease. (A) Pie charts showing patient's distribution into groups. (B) Timeline of Shannon diversity changes in patients suffering from SkAE. The particular time of SkAE manifestation is color-coded in red. This graphs only show a shift of Shannon entropy in particular patient's visits. (C) Principal coordinate analysis of the Bray-Curtis distance between samples from patients with SkAE. All samples from one patient are represented by the same symbol, and the order of sampling is indicated by a label in italics, i.e., $I$ is the first sampling. SkAE manifestation is color-coded in red. Groups were compared by PERMANOVA. (D) Relative abundances of the most abundant taxa of skin microbiota at the genus and species level in patients with SkAE. Visit number, response to treatment, and the disease index for CD (HBI) or UC (pMayo) are shown. In all panels: *p<0.05; ** $<0.01$; ** $\mathrm{p}<0.001$. P7, P21, P27, P53, P6, P20, P25, and P26 represent the patient's code designations. CD, Crohn's disease; UC, ulcerative colitis. Response: B, baseline; P, partial; F, full; N, no response. HBI, Harvey-Bradshaw index. pMayo, partial Mayo score.

\section{Figure 3}

See image above for figure legend. 
Figure 4

A
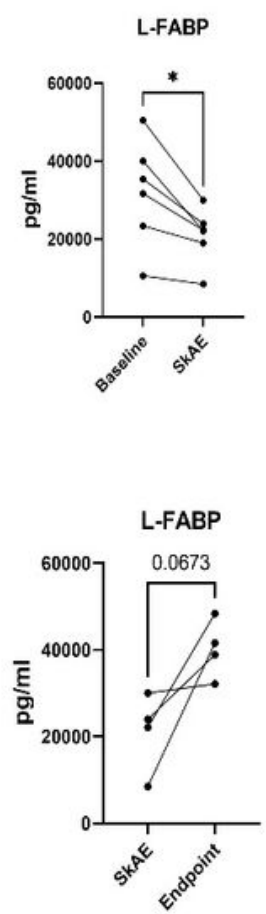

I-FABP

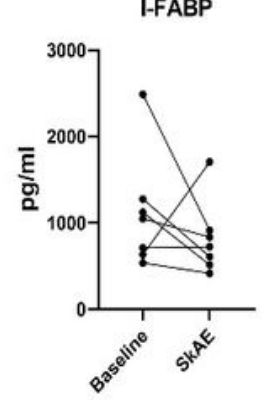

I-FABP

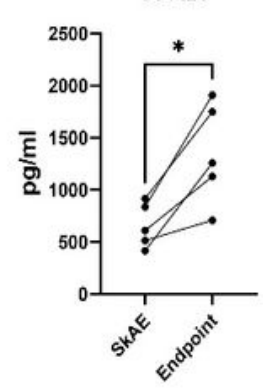

E-FABP
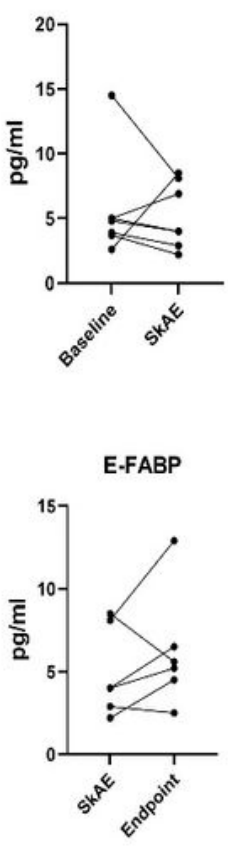

B
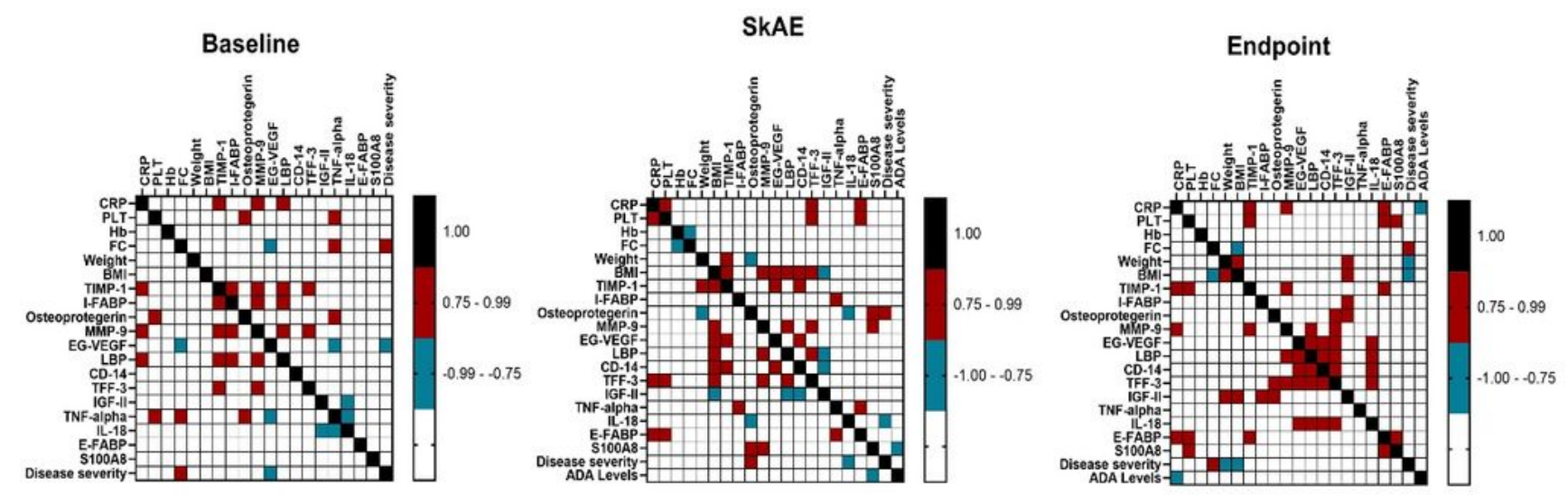

The analysis of I-FABP, L-FABP, and E-FABP in serum of patients with SkAE. (A) Comparison of L-FABP, IFABP, and E-FABP levels in patients $(n=7)$ at baseline, during the manifestation of SkAE and at the endpoint. For all panels: $(* \mathrm{p}<0.05$; paired t-test). (B) Correlation heatmap showing the Spearman's correlation coefficient of pairwise comparison between clinical parameters and biomarkers at baseline, during SkAE incidence, and at the study endpoint (burgundy, positive correlation; turquoise, negative correlation). The heatmap was constructed in GraphPad Prism, version 8.4.3.

\section{Figure 4}

See image above for figure legend.

\section{Supplementary Files}

This is a list of supplementary files associated with this preprint. Click to download. 
- STEHLIKOVASkAESupplementaryfile02032022.docx 